\title{
Generalizations of some integral inequalities related to Hardy type integral inequalities via $(p, q)$-calculus
}

\author{
Suriyakamol Thongjob ${ }^{1}$ (D), Kamsing Nonlaopon ${ }^{1 *}$ (D), Jessada Tariboon ${ }^{2}$ (D) and Sortiris K. Ntouyas ${ }^{3,4}$ (D)
}

"Correspondence: nkamsi@kku.ac.th ${ }^{1}$ Department of Mathematics, Khon Kaen University, 40002, Khon Kaen, Thailand

Full list of author information is available at the end of the article

\begin{abstract}
In this paper, we study generalizations of some integral inequalities related to Hardy type integral inequalities via $(p, q)$-calculus. Many results obtained in this paper provide extensions of existing results in the literature. Furthermore, some examples are given to illustrate the investigated results.
\end{abstract}

MSC: 05A30; 26D10; 26D15; 26A33

Keywords: Hardy type integral inequalities; $(p, q)$-calculus; $(p, q)$-differentiable function; $(p, q)$-integrable function

\section{Introduction}

Quantum calculus, shortly $q$-calculus, is the study of calculus without limits. The history of $q$-calculus can be dated back to Euler, who first introduced $q$-calculus in the track of Newton's work on infinite series. In 1910, F. H. Jackson [1] defined the definite $q$-integral, which is known as the $q$-Jackson integral. It was the starting point of $q$-calculus in a systematic way. In recent years, $q$-calculus has been actively developed and many researchers have been increasingly interested in the topic of $q$-calculus due to applications of the $q$ calculus in mathematics and physics such as combinatorics, dynamical systems, fractals, number theory, orthogonal polynomials, special functions, mechanics and also for scientific problems in some applied areas, see [2-16] for more details.

The subject $(p, q)$-calculus is a generalization of $q$-calculus and it is two parameters quantum calculus. In 2013, P.N. Sadjang [17] studied the $(p, q)$-derivative, the $(p, q)$ integral, and obtained some of their properties and the fundamental of $(p, q)$-calculus. Recently, M. Tunç, and E. Göv [18] defined the $(p, q)$-derivative and $(p, q)$-integral on finite interval. The applications of $(p, q)$-calculus play important roles in physical sciences, number theory, orthogonal polynomials, see [19-23] for more details. Furthermore, they studied some properties of $(p, q)$-calculus and $(p, q)$-analogue of some important integral inequalities. The $(p, q)$-integral inequalities have been studied and rapidly developed during this period by many authors, see [24-31] and the references therein.

Mathematical inequalities have been applied in various branches of mathematics like analysis, differential equations, and geometry. One of the famous inequalities is the Hardy

(c) The Author(s) 2021. This article is licensed under a Creative Commons Attribution 4.0 International License, which permits use sharing, adaptation, distribution and reproduction in any medium or format, as long as you give appropriate credit to the original author(s) and the source, provide a link to the Creative Commons licence, and indicate if changes were made. The images or other third party material in this article are included in the article's Creative Commons licence, unless indicated otherwise in a credit line to the material. If material is not included in the article's Creative Commons licence and your intended use is not permitted by statutory regulation or exceeds the permitted use, you will need to obtain permission directly from the copyright holder. To view a copy of this licence, visit http://creativecommons.org/licenses/by/4.0/. 
inequality. Let us just mention that in 1920, G. H. Hardy [32] presented the following famous inequality for $f$ being a non-negative integrable function and $s>1$ :

$$
\int_{0}^{\infty}\left(\frac{1}{x} \int_{0}^{x} f(t) d t\right)^{s} d x \leq\left(\frac{s}{s-1}\right)^{s} \int_{0}^{\infty} f^{s}(x) d x
$$

which is now known as Hardy inequality. This inequality plays an important role in analysis and applications, see [33, 34] for more details.

The Hardy inequality has been studied by a large number of authors during the twentieth century. Over the last 20 years, a large number of papers have appeared in the literature which deals with the simple proofs, various generalizations and discrete analogue of Hardy inequality, see [35-39] for more details.

In 2014, L. Maligranda et al. [40] studied a $q$-analogue of Hardy inequality (1.1) and some related inequalities. It seems to be a huge new research area to study of these so called $q$-Hardy type inequalities. They obtained more general results on $q$-Hardy type inequalities. By taking $q \rightarrow 1$, we obtain classical results on Hardy inequality (1.1). Next, L.-E. Persson and S. Shaimardan [41] studied some $q$-analogue of Hardy type inequalities for the Riemann-Liouville fractional integral operator; see [42, 43] for more details.

In 1964, N. Levinson [44] presented the inequality respecting integration from $a$ to $b$ for $0<a<b<\infty, f$ is a non-negative integrable function and $s>1$, then

$$
\int_{a}^{b}\left(\frac{1}{x} \int_{a}^{x} f(t) d t\right)^{s} d x \leq\left(\frac{s}{s-1}\right)^{s} \int_{a}^{b} f^{s}(x) d x
$$

In 2012, W.T. Sulaiman [45] gave a generalization and improvement for inequalities similar to Hardy inequality in the sense when $f>0$ on $[a, b] \subset(0, \infty)$ and $0<k<1 \leq h$, as follows:

$$
h \int_{a}^{b} \frac{1}{x^{h}}\left(\int_{a}^{x} f(t) d t\right)^{h} d x \leq(b-a)^{h} \int_{a}^{b}\left(\frac{f(x)}{x}\right)^{h} d x-\int_{a}^{b} \frac{(x-a)^{h}}{x^{h}} f^{h}(x) d x
$$

and

$$
k \int_{a}^{b} \frac{1}{x^{k}}\left(\int_{a}^{x} f(t) d t\right)^{k} d x \leq(1-a / b)^{k} \int_{a}^{b} f^{k}(x) d x-\frac{1}{b^{k}} \int_{a}^{b}(x-a)^{k} f^{k}(x) d x .
$$

In 2013, B. Sroysang [46] presented a generalization for inequalities (1.3) and (1.4) with additional parameter $m$ in the sense when $f>0$ on $[a, b] \subset(0, \infty), 0<k<1 \leq h$ and $m>0$, as follows:

$$
h \int_{a}^{b} \frac{1}{x^{m}}\left(\int_{a}^{x} f(t) d t\right)^{h} d x \leq(b-a)^{h} \int_{a}^{b} \frac{f^{h}(x)}{x^{m}} d x-\int_{a}^{b} \frac{(x-a)^{h}}{x^{m}} f^{h}(x) d x
$$

and

$$
k \int_{a}^{b} \frac{1}{x^{m}}\left(\int_{a}^{x} f(t) d t\right)^{k} d x \leq \frac{(b-a)^{k}}{b^{m}} \int_{a}^{b} f^{k}(x) d x-\frac{1}{b^{m}} \int_{a}^{b}(x-a)^{k} f^{k}(x) d x .
$$

In 2014, B. Sroysang [47] presented a new kind of Hardy inequality and obtained a direct generalization of the original Hardy inequality. Next, K. Mehrez [48] studied some 
generalizations and new refined Hardy type inequalities by using Jensen's inequality and Chebyshev integral inequality, see [49-52] for more details.

In 2016, S. Wu, B. Sroysang and S. Li [53] investigated certain integral inequalities similar to the Hardy inequality. They generalized versions of some known results related to the Hardy inequality and gave some new integral inequalities of Hardy type by introducing a monotonous function and established the inequality for $\beta$ being a non-negative real number, as follows:

$$
\int_{a}^{b}\left(\frac{F(x)}{g(x)}\right)^{p} d x \leq\left(\frac{p}{p-1}\right)^{p} \int_{a}^{b}\left((x-a+\beta) \frac{f(x)}{g(x)}\right)^{p} d x
$$

where $f \geq 0, g>0$ on $[a, b] \subseteq(0, \infty), p \geq 1,(x-a+\beta) / g(x)$ is non-increasing, and

$$
F(x)=\int_{a}^{x} f(t) d t
$$

for $x \in[a, b]$.

Inspired by this ongoing study, we establish the generalization of some integral inequalities related to Hardy type integral inequalities via $(p, q)$-calculus. Many results obtained in this paper provide extensions of other results given in previous papers. Furthermore, we give some examples to illustrate the investigated results.

\section{Preliminaries}

Throughout this paper, let $[a, b] \subseteq \mathbb{R}$ be an interval and $0<q<p \leq 1$. The following definitions and theorems for $(p, q)$-calculus are given in [17, 18, 24-29].

First, we give some $(p, q)$-notation, which would appear in this paper. For any real number $n$, the $(p, q)$-analogue of $n$ is defined by

$$
[n]_{p, q}=\frac{p^{n}-q^{n}}{p-q}
$$

and

$$
\begin{gathered}
{[-n]_{p, q}=-\frac{1}{(p q)^{n}}[n]_{p, q} .} \\
\text { If } p=1 \text {, then }(2.1) \text { reduces to } \\
{[n]_{q}=\frac{1-q^{n}}{1-q},}
\end{gathered}
$$

which is $q$-analogue of $n$.

Definition 2.1 ([24]) If $f:[a, b] \rightarrow \mathbb{R}$ is a continuous function, then the $(p, q)$-derivative of the function $f$ on $[a, b]$ at $x$ is defined by

$$
{ }_{a} D_{p, q} f(x)=\frac{f(p x+(1-p) a)-f(q x+(1-q) a)}{(p-q)(x-a)}, \quad x \neq a .
$$

The function $f$ is said to be a $(p, q)$-differentiable function on $[a, b]$ if ${ }_{a} D_{p, q} f(x)$ exists for all $x \in[a, b]$. 
It should be noted that

$$
{ }_{a} D_{p, q} f(a)=\lim _{x \rightarrow a} D_{p, q} f(x) .
$$

In Definition 2.1, if $a=0$, then ${ }_{0} D_{p, q} f=D_{p, q} f$ is defined by

$$
D_{p, q} f(x)=\frac{f(p x)-f(q x)}{(p-q) x}, x \neq 0 .
$$

And, if $p=1$, then $D_{p, q} f(x)=D_{q} f(x)$, which is the $q$-derivative of the function $f$, and also if $q \rightarrow 1$ in (2.5), then it reduces to a classical derivative.

Example 2.1 Define function $f:[a, b] \rightarrow \mathbb{R}$ by $f(x)=2 x^{2}+2 x+c$, where $c$ is a constant. Then, for $x \neq a$, we have

$$
\begin{aligned}
{ }_{a} D_{p, q}\left(2 x^{2}+2 x+c\right)= & \frac{2[p x+(1-p) a]^{2}+2[p x+(1-p) a]+c}{(p-q)(x-a)} \\
& -\frac{2[q x+(1-q) a]^{2}+2[q x+(1-q) a]+c}{(p-q)(x-a)} \\
& =\frac{2(p+q) x^{2}-4 a x(p+q)+4 a x-4 a^{2}+2 a^{2}(p+q)+2(x-a)}{x-a} \\
& =2(p+q)(x-a)+4 a+2 .
\end{aligned}
$$

Theorem 2.1 Iff, $g:[a, b] \rightarrow \mathbb{R}$ are continuous functions and $c, d$ are constants, then the following formulas hold:

(i) ${ }_{a} D_{p, q}[c f(x) \pm d g(x)]=c_{a} D_{p, q} f(x) \pm d_{a} D_{p, q} g(x)$;

(ii) ${ }_{a} D_{p, q}[f(x) g(x)]=f(p x+(1-p) a)_{a} D_{p, q} g(x)+g(q x+(1-q) a)_{a} D_{p, q} f(x)$;

(iii) ${ }_{a} D_{p, q}\left[\frac{f(x)}{g(x)}\right]=\frac{g(p x+(1-p) a)_{a} D_{p, q} f(x)-f(p x+(1-p) a)_{a} D_{p, q} g(x)}{g(p x+(1-p) a) g(q x+(1-q) a)}$.

The proof of this theorem is given by [18].

Definition 2.2 ([24]) If $f:[a, b] \rightarrow \mathbb{R}$ is a continuous function and $0<a<b$, then the $(p, q)$-integral is defined by

$$
\int_{a}^{b} f(x)_{a} d_{p, q} x=(p-q)(b-a) \sum_{k=0}^{\infty} \frac{q^{k}}{p^{k+1}} f\left(\frac{q^{k}}{p^{k+1}} b+\left(1-\frac{q^{k}}{p^{k+1}}\right) a\right) .
$$

$f$ is said to be a $(p, q)$-integrable function on $[a, b]$ if $\int_{a}^{b} f(x)_{a} d_{p, q} x$ exists for all $x \in[a, b]$.

Moreover, if $c \in(a, b)$, then $(p, q)$-integral is defined by

$$
\int_{c}^{b} f(x)_{a} d_{p, q} x=\int_{a}^{b} f(x)_{a} d_{p, q} x-\int_{a}^{c} f(x)_{a} d_{p, q} x .
$$

If $a=0$ in (2.6), then one can get the classical $(p, q)$-integral defined by

$$
\int_{0}^{b} f(x) d_{p, q} x=(p-q) b \sum_{k=0}^{\infty} \frac{q^{k}}{p^{k+1}} f\left(\frac{q^{k}}{p^{k+1}} b\right)
$$


and

$$
\int_{a}^{b} f(x) d_{p, q} x=\int_{0}^{b} f(x) d_{p, q} x-\int_{0}^{a} f(x) d_{p, q} x .
$$

Example 2.2 Define a function $f:[a, b] \rightarrow \mathbb{R}$ by $f(x)=x^{2}+2 x+c$, where $c$ is a constant. Then we have

$$
\begin{aligned}
\int_{a}^{b} f(x)_{a} d_{p, q} x= & \int_{a}^{b}\left(x^{2}+2 x+c\right)_{a} d_{p, q} x \\
= & (p-q)(b-a) \sum_{k=0}^{\infty} \frac{q^{k}}{p^{k+1}}\left(\frac{q^{k}}{p^{k+1}} b+\left(1-\frac{q^{k}}{p^{k+1}}\right) a\right)^{2} \\
& +2(p-q)(b-a) \sum_{k=0}^{\infty} \frac{q^{k}}{p^{k+1}}\left(\frac{q^{k}}{p^{k+1}} b+\left(1-\frac{q^{k}}{p^{k+1}}\right) a\right) \\
& +(p-q)(b-a) \sum_{k=0}^{\infty} \frac{q^{k}}{p^{k+1}}(c) \\
= & \frac{(b-a)^{3}}{[3]_{p, q}}+\frac{2(b-a)[a(b-a)+b-a(1-p-q)]}{[2]_{p, q}}+(b-a)\left(a^{2}+c\right) \\
= & \frac{(b-a)^{3}}{[3]_{p, q}}+\frac{2(a+1)(b-a)^{2}}{[2]_{p, q}}+(b-a)\left(a^{2}+2 a+c\right) .
\end{aligned}
$$

The proofs of the following theorems are given in [18].

Theorem 2.2 If $f, g:[a, b] \rightarrow \mathbb{R}$ are continuous functions, $t \in[a, b]$ and $\alpha$ is a constant, then the following formulas hold:

(i) ${ }_{a} D_{p, q} \int_{a}^{t} f(x)_{a} d_{p, q} x=f(t)$;

(ii) $\int_{c}^{t}{ }_{a} D_{p, q} f(x)_{a} d_{p, q} x=f(t)-f(c)$ for $c \in(a, t)$;

(iii) $\int_{a}^{t}[f(x)+g(x)]_{a} d_{p, q} x=\int_{a}^{b} f(x)_{a} d_{p, q} x+\int_{a}^{b} g(x)_{a} d_{p, q} x$;

(iv) $\int_{a}^{t} \alpha f(x)_{a} d_{p, q} x=\alpha \int_{a}^{b} f(x)_{a} d_{p, q} x$;

(v) $\int_{a}^{t}(x-a)^{\alpha}{ }_{a} d_{p, q} x=\frac{(t-a)^{\alpha+1}}{[\alpha+1]_{p, q}}$;

(vi) $\int_{c}^{t} f(p x+(1-p) a)_{a} D_{p, q} g(x)_{a} d_{p, q} x=\left.(f g)(x)\right|_{c} ^{t}-\int_{c}^{t} g(q x+(1-q) a)_{a} D_{p, q} f(x)_{a} d_{p, q} x$.

Theorem 2.3 Iff, $g:[a, b] \rightarrow \mathbb{R}$ are continuous functions and $r>1$ with $1 / r+1 / s=1$, then

$$
\int_{a}^{b}|f(x) g(x)|_{a} d_{p, q} x \leq\left(\int_{a}^{b}|f(x)|^{r}{ }_{a} d_{p, q} x\right)^{1 / r}\left(\int_{a}^{b}|g(x)|^{s}{ }_{a} d_{p, q} x\right)^{1 / s}
$$

\section{Main results}

In this section, we are going to establish the generalization of some integral inequalities related to Hardy type integral inequalities via $(p, q)$-calculus. The first result is presented as follows.

Theorem 3.1 If $f, g:[a, b] \rightarrow \mathbb{R}$ are positive continuous functions and $0<r<1$ with $1 / r+$ $1 / s=1$, then

$$
\int_{a}^{b}|f(x) g(x)|_{a} d_{p, q} x \geq\left(\int_{a}^{b}|f(x)|^{r}{ }_{a} d_{p, q} x\right)^{1 / r}\left(\int_{a}^{b}|g(x)|^{s}{ }_{a} d_{p, q} x\right)^{1 / s} .
$$


Proof From Theorem 2.3, we get

$$
\begin{aligned}
\int_{a}^{b}|f(x)|_{a}^{r} d_{p, q} x & \leq\left(\int_{a}^{b}\left(|f(x) g(x)|^{1 / k}\right)^{k}{ }_{a} d_{p, q} x\right)^{1 / k}\left(\int_{a}^{b}\left(|g(x)|^{-1 / k}\right)^{k^{\prime}}{ }_{a} d_{p, q} x\right)^{1 / k^{\prime}} \\
& =\left(\int_{a}^{b}|f(x) g(x)|_{a} d_{p, q} x\right)^{1 / k}\left(\int_{a}^{b}|g(x)|^{-k^{\prime} / k}{ }_{a} d_{p, q} x\right)^{1 / k^{\prime}}
\end{aligned}
$$

where $r=1 / k$ and $1 / k+1 / k^{\prime}=1$.

Consequently,

$$
\left(\int_{a}^{b}|f(x)|_{a}^{r} d_{p, q} x\right)^{1 / r} \leq\left(\int_{a}^{b}|f(x) g(x)|_{a} d_{p, q} x\right)^{1 /(r k)}\left(\int_{a}^{b}|g(x)|^{-k^{\prime} / k}{ }_{a} d_{p, q} x\right)^{1 /\left(r k^{\prime}\right)},
$$

or

$$
\left(\int_{a}^{b}|f(x)|_{a}^{r} d_{p, q} x\right)^{1 / r}\left(\int_{a}^{b}|g(x)|^{-k^{\prime} / k}{ }_{a} d_{p, q} x\right)^{-1 /\left(r k^{\prime}\right)} \leq \int_{a}^{b}|f(x) g(x)|_{a} d_{p, q} x .
$$

Therefore,

$$
\int_{a}^{b}|f(x) g(x)|_{a} d_{p, q} x \geq\left(\int_{a}^{b}|f(x)|^{r}{ }_{a} d_{p, q} x\right)^{1 / r}\left(\int_{a}^{b}|g(x)|^{s}{ }_{a} d_{p, q} x\right)^{1 / s} .
$$

The proof is thus accomplished.

Theorem 3.2 Iff is a non-negative function, $g$ is a positive function on $[a, b] \subseteq(0, \infty), \gamma$ is a positive real number, $r>1$, and $(x-a+\gamma) / g(x)$ is non-increasing, then

$$
\int_{a}^{b} \frac{1}{g^{r}(x)}\left(\int_{a}^{x} f(t)_{a} d_{p, q} t\right)^{r}{ }_{a} d_{p, q} x \leq \frac{(p q)^{1-1 / r}}{[1-1 / r]_{p, q}^{r}} \int_{a}^{b} \frac{(x-a+\gamma)^{r}}{g^{r}(x)} f^{r}(x)_{a} d_{p, q} x .
$$

Proof From Theorem 2.3, we get

$$
\begin{aligned}
\int_{a}^{b} \frac{1}{g^{r}(x)}\left(\int_{a}^{x} f(t)_{a} d_{p, q} t\right)^{r}{ }_{a} d_{p, q} x \leq & \int_{a}^{b} g^{-r}(x) \int_{a}^{x}(t-a)^{1-1 / r} f^{r}(t)_{a} d_{p, q} t \\
& \times\left(\int_{a}^{x}(t-a)^{-1 / r} d_{p, q} t\right)^{r-1}{ }_{a} d_{p, q} x .
\end{aligned}
$$

By Theorem 2.2(v), we have

$$
\begin{aligned}
& \int_{a}^{b} \frac{1}{g^{r}(x)}\left(\int_{a}^{x} f(t)_{a} d_{p, q} t\right)^{r} d_{p, q} x \\
& \quad \leq \frac{1}{[1-1 / r]_{p, q}^{r-1}} \int_{a}^{b} g^{-r}(x) \int_{a}^{x} f^{r}(t)(t-a)^{1-1 / r}(x-a)^{(1-1 / r)(r-1)}{ }_{a} d_{p, q} t_{a} d_{p, q} x \\
& \quad=\frac{1}{[1-1 / r]_{p, q}^{r-1}} \int_{a}^{b} \int_{t}^{b} g^{-r}(x) f^{r}(t)(t-a)^{1-1 / r}(x-a)^{(1-1 / r)(r-1)}{ }_{a} d_{p, q} x_{a} d_{p, q} t \\
& \quad=\frac{1}{[1-1 / r]_{p, q}^{r-1}} \int_{a}^{b} \int_{t}^{b} \frac{(x-a)^{r}}{g^{r}(x)} f^{r}(t)(t-a)^{1-1 / r}(x-a)^{1 / r-2}{ }_{a} d_{p, q} x_{a} d_{p, q} t
\end{aligned}
$$




$$
\leq \frac{1}{[1-1 / r]_{p, q}^{r-1}} \int_{a}^{b} \int_{t}^{b} \frac{(x-a+\gamma)^{r}}{g^{r}(x)} f^{r}(t)(t-a)^{1-1 / r}(x-a)^{1 / r-2}{ }_{a} d_{p, q} x_{a} d_{p, q} t
$$

By the assumption that the function $(x-a+\gamma) / g(x)$ is non-increasing and Theorem 2.2(v), we have

$$
\begin{aligned}
& \int_{a}^{b} \frac{1}{g^{r}(x)}\left(\int_{a}^{x} f(t)_{a} d_{p, q} t\right)^{r}{ }_{a} d_{p, q} x \\
& \quad \leq \frac{1}{[1-1 / r]_{p, q}^{r-1}} \int_{a}^{b} \frac{(t-a+\gamma)^{r}}{g^{r}(t)} f^{r}(t)(t-a)^{1-1 / r} \int_{t}^{b}(x-a)^{1 / r-2}{ }_{a} d_{p, q} x_{a} d_{p, q} t \\
& \quad=\frac{1}{[1-1 / r]_{p, q}^{r-1}} \int_{a}^{b} \frac{(t-a+\gamma)^{r}}{g^{r}(t)} f^{r}(t)\left(\frac{(b-a)^{1 / r-1}-(t-a)^{1 / r-1}}{[1 / r-1]_{p, q}}\right)(t-a)^{1-1 / r}{ }_{a} d_{p, q} t \\
& \quad=\frac{(p q)^{1-1 / r}}{[1-1 / r]_{p, q}^{r}} \int_{a}^{b} \frac{(t-a+\gamma)^{r}}{g^{r}(t)} f^{r}(t)\left(1-\frac{(t-a)^{1-1 / r}}{(b-a)^{1-1 / r}}\right) a d_{p, q} t \\
& \quad \leq \frac{(p q)^{1-1 / r}}{[1-1 / r]_{p, q}^{r}} \int_{a}^{b} \frac{(t-a+\gamma)^{r}}{g^{r}(t)} f^{r}(t)_{a} d_{p, q} t .
\end{aligned}
$$

This proof is completed.

Corollary 3.1 Iff is a non-negative function, $\gamma$ is a positive real number, and $r>1$, then

$$
\int_{a}^{b} \frac{1}{(x-a+\gamma)^{r}}\left(\int_{a}^{x} f(t)_{a} d_{p, q} t\right)_{a}^{r} d_{p, q} x \leq \frac{(p q)^{1-1 / r}}{[1-1 / r]_{p, q}^{r}} \int_{a}^{b} f^{r}(x)_{a} d_{p, q} x
$$

Remark 3.1 (1) If $p=1$, then (3.2) reduces to

$$
\int_{a}^{b} \frac{1}{g^{r}(x)}\left(\int_{a}^{x} f(t)_{a} d_{q} t\right)^{r} d_{q} x \leq \frac{q^{1-1 / r}}{[1-1 / r]_{q}^{r}} \int_{a}^{b} \frac{(x-a+\gamma)^{r}}{g^{r}(x)} f^{r}(x)_{a} d_{q} x
$$

Also, if $q \rightarrow 1$, then (3.4) reduces to an inequality, which appeared in [53].

(2) If $0<\gamma<a$, we obtain the following inequality:

$$
\begin{aligned}
\int_{a}^{b} \frac{1}{x^{r}}\left(\int_{a}^{x} f(t)_{a} d_{p, q} t\right)^{r}{ }_{a} d_{p, q} x & \leq \int_{a}^{b} \frac{1}{(x-a+\gamma)^{r}}\left(\int_{a}^{x} f(t)_{a} d_{p, q} t\right)^{r}{ }_{a} d_{p, q} x \\
& \leq \frac{(p q)^{1-1 / r}}{[1-1 / r]_{p, q}^{r}} \int_{a}^{b} f^{r}(x)_{a} d_{p, q} x .
\end{aligned}
$$

Also, if $p=1$ and $q \rightarrow 1$, then (3.5) is reduced to (1.2).

Theorem 3.3 Iff is a non-negative function, $g$ is a positive function on $[a, b] \subseteq(0, \infty), \gamma$ is a positive real number, $r>1,0 \leq r-m<1-1 / r$, and $(x-a+\gamma) / g(x)$ is non-increasing, then

$$
\begin{aligned}
\int_{a}^{b} \frac{1}{g^{m}(x)}\left(\int_{a}^{x} f(t)_{a} d_{p, q} t\right)^{r} d_{p, q} x \leq & \frac{(p q)^{m+1-r-1 / r}}{[1-1 / r]_{p, q}^{r-1}[m+1-r-1 / r]_{p, q}} \\
& \times \int_{a}^{b} \frac{(x-a+\gamma)^{r}}{g^{m}(x)} f^{r}(x)_{a} d_{p, q} x
\end{aligned}
$$


Proof From Theorem 2.3, we get

$$
\begin{aligned}
\int_{a}^{b} \frac{1}{g^{m}(x)}\left(\int_{a}^{x} f(t)_{a} d_{p, q} t\right)^{r}{ }_{a} d_{p, q} x \leq & \int_{a}^{b} g^{-m}(x) \int_{a}^{x}(t-a)^{1-1 / r} f^{r}(t)_{a} d_{p, q} t \\
& \times\left(\int_{a}^{x}(t-a)^{-1 / r} d_{p, q} t\right)^{r-1}{ }_{a} d_{p, q} x .
\end{aligned}
$$

By Theorem 2.2(v), we have

$$
\begin{aligned}
& \int_{a}^{b} \frac{1}{g^{m}(x)}\left(\int_{a}^{x} f(t)_{a} d_{p, q} t\right)_{a}^{r} d_{p, q} x \\
& \leq \frac{1}{[1-1 / r]_{p, q}^{r-1}} \int_{a}^{b} g^{-m}(x) \int_{a}^{x} f^{r}(t)(t-a)^{1-1 / r}(x-a)^{(1-1 / r)(r-1)}{ }_{a} d_{p, q} t_{a} d_{p, q} x \\
& \quad=\frac{1}{[1-1 / r]_{p, q}^{r-1}} \int_{a}^{b} \int_{t}^{b} g^{-m}(x) f^{r}(t)(t-a)^{1-1 / r}(x-a)^{(1-1 / r)(r-1)}{ }_{a} d_{p, q} x_{a} d_{p, q} t \\
& \quad=\frac{1}{[1-1 / r]_{p, q}^{r-1}} \int_{a}^{b} \int_{t}^{b} \frac{(x-a)^{m}}{g^{m}(x)} f^{r}(t)(t-a)^{1-1 / r}(x-a)^{r+1 / r-2-m}{ }_{a} d_{p, q} x_{a} d_{p, q} t \\
& \leq \frac{1}{[1-1 / r]_{p, q}^{r-1}} \int_{a}^{b} \int_{t}^{b} \frac{(x-a+\gamma)^{m}}{g^{m}(x)} f^{r}(t)(t-a)^{1-1 / r}(x-a)^{r+1 / r-2-m}{ }_{a} d_{p, q} x_{a} d_{p, q} t .
\end{aligned}
$$

By the assumption that the function $(x-a+\gamma) / g(x)$ is non-increasing and Theorem 2.2(v), we have

$$
\begin{aligned}
\int_{a}^{b} & \frac{1}{g^{r}(x)}\left(\int_{a}^{x} f(t)_{a} d_{p, q} t\right)_{a}^{r} d_{p, q} x \\
\leq & \frac{1}{[1-1 / r]_{p, q}^{r-1}} \int_{a}^{b} \frac{(t-a+\gamma)^{m}}{g^{m}(t)} f^{r}(t)(t-a)^{1-1 / r} \int_{t}^{b}(x-a)^{r+1 / r-2-m}{ }_{a} d_{p, q} x_{a} d_{p, q} t \\
= & \frac{1}{[1-1 / r]_{p, q}^{r-1}} \int_{a}^{b} \frac{(t-a+\gamma)^{m}}{g^{m}(t)} f^{r}(t) \\
= & \frac{\left(\frac{(b-a)^{r+1 / r-1-m}-(t-a)^{r+1 / r-1-m}}{[r+1 / r-1-m]_{p, q}}\right) \times(t-a)^{1-1 / r}{ }_{a} d_{p, q} t}{[1-1 / r]_{p, q}^{r-1}[m+1-r-1 / r]_{p, q}} \int_{a}^{b} \frac{(t-a+\gamma)^{m}}{g^{m}(t)} f^{r}(t) \\
& \times\left((t-a)^{r-m}-\frac{(t-a)^{1-1 / r}}{(b-a)^{1-1 / r+m-r}}\right)_{a} d_{p, q} t \\
\leq & \frac{(p q)^{m+1-r-1 / r}}{[1-1 / r]_{p, q}^{r-1}[m+1-r-1 / r]_{p, q}} \int_{a}^{b} \frac{(t-a+\gamma)^{m}}{g^{m}(t)}(t-a+\gamma)^{r-m} f^{r}(t)_{a} d_{p, q} t \\
= & \frac{(p q)^{m+1-r-1 / r}}{[1-1 / r]_{p, q}^{r-1}[m+1-r-1 / r]_{p, q}} \int_{a}^{b} \frac{(t-a+\gamma)^{r}}{g^{m}(t)} f^{r}(t)_{a} d_{p, q} t .
\end{aligned}
$$

Hence, the inequality (3.6) is established. 
Remark 3.2 If $p=1$, then (3.6) reduces to a generalization of $q$-Hardy inequality as

$$
\begin{aligned}
\int_{a}^{b} \frac{1}{g^{m}(x)}\left(\int_{a}^{x} f(t)_{a} d_{q} t\right)^{r}{ }_{a} d_{q} x \leq & \frac{q^{m+1-r-1 / r}}{[1-1 / r]_{q}^{r-1}[m+1-r-1 / r]_{q}} \\
& \times \int_{a}^{b} \frac{(x-a+\gamma)^{r}}{g^{m}(x)} f^{r}(x)_{a} d_{q} x
\end{aligned}
$$

Theorem 3.4 Iff is a positive function on $[a, b] \subseteq(0, \infty), r \geq 1$, and $m>0$, then

$$
\begin{aligned}
{[r]_{p, q} \int_{a}^{b} \frac{1}{x^{m}}\left(\int_{a}^{x} f(t)_{a} d_{p, q} t\right)^{r} d_{p, q} x \leq } & (b-a)^{r} \int_{a}^{b} \frac{f^{r}(x)}{x^{m}}{ }_{a} d_{p, q} x \\
& -\int_{a}^{b} \frac{(x-a)^{r}}{x^{m}} f^{r}(x)_{a} d_{p, q} x
\end{aligned}
$$

Proof From Theorem 2.3, we get

$$
\begin{aligned}
\int_{a}^{b} & \frac{1}{x^{m}}\left(\int_{a}^{x} f(t)_{a} d_{p, q} t\right)_{a}^{r} d_{p, q} x \\
\leq & \int_{a}^{b} x^{-m}\left(\int_{a}^{x} f^{r}(t)_{a} d_{p, q} t\right)\left(\int_{a}^{x}{ }_{a} d_{p, q} t\right)^{r-1}{ }_{a} d_{p, q} x \\
& =\int_{a}^{b} x^{-m}\left(\int_{a}^{x} f^{r}(t)_{a} d_{p, q} t\right)(x-a)^{r-1}{ }_{a} d_{p, q} x \\
& =\int_{a}^{b} \int_{t}^{b} x^{-m} f^{r}(t)(x-a)^{r-1}{ }_{a} d_{p, q} x_{a} d_{p, q} t \\
& \leq \int_{a}^{b} t^{-m} f^{r}(t)\left(\int_{t}^{b}(x-a)^{r-1}{ }_{a} d_{p, q} x\right){ }_{a} d_{p, q} t .
\end{aligned}
$$

By Theorem 2.2(v), we obtain

$$
\begin{aligned}
& \int_{a}^{b} \frac{1}{x^{m}}\left(\int_{a}^{x} f(t)_{a} d_{p, q} t\right)^{r}{ }_{a} d_{p, q} x \\
& =\int_{a}^{b} t^{-m} f^{r}(t)\left(\frac{(b-a)^{r}-(t-a)^{r}}{[r]_{p, q}}\right){ }_{a} d_{p, q} t \\
& =\frac{1}{[r]_{p, q}}\left((b-a)^{r} \int_{a}^{b} \frac{f^{r}(t)}{t^{m}}{ }_{a} d_{p, q} t-\int_{a}^{b} \frac{(t-a)^{r}}{t^{m}} f^{r}(t)_{a} d_{p, q} t\right) .
\end{aligned}
$$

The inequality (3.8) is proved.

Remark 3.3 If $p=1$, then (3.8) reduces to

$$
\begin{aligned}
& {[r]_{q} \int_{a}^{b} \frac{1}{x^{m}}\left(\int_{a}^{x} f(t)_{a} d_{q} t\right)^{r}{ }_{a} d_{q} x} \\
& \quad \leq(b-a)^{r} \int_{a}^{b} \frac{f^{r}(x)}{x^{m}} a_{q} x-\int_{a}^{b} \frac{(x-a)^{r}}{x^{m}} f^{r}(x)_{a} d_{q} x .
\end{aligned}
$$

Also, if $q \rightarrow 1$, then (3.9) reduces to an inequality, which appeared in [46]. 
Theorem 3.5 Iff is a positive function on $[a, b] \subseteq(0, \infty), 0<r<1$, and $m>0$, then

$$
\begin{aligned}
{[r]_{p, q} \int_{a}^{b} \frac{1}{x^{m}}\left(\int_{a}^{x} f(t)_{a} d_{p, q} t\right)^{r}{ }_{a} d_{p, q} x } & \geq \frac{(b-a)^{r}}{b^{m}} \int_{a}^{b} f^{r}(x)_{a} d_{p, q} x \\
& -\frac{1}{b^{m}} \int_{a}^{b}(x-a)^{r} f^{r}(x)_{a} d_{p, q} x .
\end{aligned}
$$

Proof From Theorem 3.1, we get

$$
\begin{aligned}
& \int_{a}^{b} \frac{1}{x^{m}}\left(\int_{a}^{x} f(t)_{a} d_{p, q} t\right)_{a}^{r} d_{p, q} x \\
& \geq \int_{a}^{b} x^{-m}\left(\int_{a}^{x} f^{r}(t)_{a} d_{p, q} t\right)\left(\int_{a}^{x}{ }_{a} d_{p, q} t\right)^{r-1}{ }_{a} d_{p, q} x \\
& =\int_{a}^{b} x^{-m}\left(\int_{a}^{x} f^{r}(t)_{a} d_{p, q} t\right)(x-a)^{r-1}{ }_{a} d_{p, q} x \\
& =\int_{a}^{b} \int_{t}^{b} x^{-m} f^{r}(t)(x-a)^{r-1}{ }_{a} d_{p, q} x_{a} d_{p, q} t \\
& \geq b^{-m} \int_{a}^{b} f^{r}(t)\left(\int_{t}^{b}(x-a)^{r-1}{ }_{a} d_{p, q} x\right)_{a} d_{p, q} t .
\end{aligned}
$$

By Theorem 2.2(v), we obtain

$$
\begin{aligned}
& \int_{a}^{b} \frac{1}{x^{m}}\left(\int_{a}^{x} f(t)_{a} d_{p, q} t\right)_{a}^{r} d_{p, q} \\
& =b^{-m} \int_{a}^{b} f^{r}(t)\left(\frac{(b-a)^{r}-(t-a)^{r}}{[r]_{p, q}}\right){ }_{a} d_{p, q} t \\
& =\frac{(b-a)^{r}}{b^{m}} \int_{a}^{b} f^{r}(t)_{a} d_{p, q} t-\frac{1}{b^{m}} \int_{a}^{b}(t-a)^{r} f^{r}(t)_{a} d_{p, q} t .
\end{aligned}
$$

This completes the proof.

Remark 3.4 If $p=1$, then (3.10) reduces to

$$
\begin{aligned}
& {[r]_{q} \int_{a}^{b} \frac{1}{x^{m}}\left(\int_{a}^{x} f(t)_{a} d_{q} t\right)^{r}{ }_{a} d_{q} x} \\
& \quad \geq \frac{(b-a)^{r}}{b^{m}} \int_{a}^{b} f^{r}(x)_{a} d_{q} x-\frac{1}{b^{m}} \int_{a}^{b}(x-a)^{r} f^{r}(x)_{a} d_{q} x .
\end{aligned}
$$

Also, if $q \rightarrow 1$, then (3.11) reduces to an inequality, which appeared in [46].

Theorem 3.6 Iff, $g$ are positive functions on $[a, b] \subseteq(0, \infty)$ such that $g$ is non-decreasing, $r \geq 1$, and $m>0$, then

$$
\begin{aligned}
{[r]_{p, q} \int_{a}^{b} \frac{1}{g^{m}(x)}\left(\int_{a}^{x} f(t)_{a} d_{p, q} t\right)^{r}{ }_{a} d_{p, q} x \leq } & (b-a)^{r} \int_{a}^{b} \frac{f^{r}(x)}{g^{m}(x)}{ }_{a} d_{p, q} x \\
& -\int_{a}^{b} \frac{(x-a)^{r}}{g^{m}(x)} f^{r}(x)_{a} d_{p, q} x .
\end{aligned}
$$


Proof From Theorem 2.3, we get

$$
\begin{aligned}
\int_{a}^{b} & \frac{1}{g^{m}(x)}\left(\int_{a}^{x} f(t)_{a} d_{p, q} t\right)^{r}{ }_{a} d_{p, q} x \\
\leq & \int_{a}^{b} g^{-m}(x)\left(\int_{a}^{x} f^{r}(t)_{a} d_{p, q} t\right) \\
& \times\left(\int_{a}^{x}{ }_{a} d_{p, q} t\right)^{r-1}{ }_{a} d_{p, q} x \\
= & \int_{a}^{b} g^{-m}(x)\left(\int_{a}^{x} f^{r}(t)_{a} d_{p, q} t\right)(x-a)^{r-1}{ }_{a} d_{p, q} x \\
= & \int_{a}^{b} \int_{t}^{b} g^{-m}(x) f^{r}(t)(x-a)^{r-1}{ }_{a} d_{p, q} x_{a} d_{p, q} t .
\end{aligned}
$$

By the assumption of the function $g$ and Theorem 2.2(v), we obtain

$$
\begin{aligned}
& \int_{a}^{b} \frac{1}{g^{m}(x)}\left(\int_{a}^{x} f(t)_{a} d_{p, q} t\right)_{a}^{r} d_{p, q} x \\
& \quad \leq \int_{a}^{b} g^{-m}(t) f^{r}(t)\left(\int_{t}^{b}(x-a)^{r-1}{ }_{a} d_{p, q} x\right)_{a} d_{p, q} t \\
& \quad=\int_{a}^{b} g^{-m}(t) f^{r}(t)\left(\frac{(b-a)^{r}-(t-a)^{r}}{[r]_{p, q}}\right)_{a} d_{p, q} t \\
& \quad=\frac{1}{[r]_{p, q}}\left((b-a)^{r} \int_{a}^{b} \frac{f^{r}(t)}{g^{m}(t)}{ }_{a} d_{p, q} t-\int_{a}^{b} \frac{(t-a)^{r}}{g^{m}(t)} f^{r}(t)_{a} d_{p, q} t\right),
\end{aligned}
$$

which finishes the proof.

Remark 3.5 If $p=1$, then (3.12) reduces to

$$
\begin{aligned}
& {[r]_{q} \int_{a}^{b} \frac{1}{g^{m}(x)}\left(\int_{a}^{x} f(t)_{a} d_{q} t\right)^{r}{ }_{a} d_{q} x} \\
& \quad \leq(b-a)^{r} \int_{a}^{b} \frac{f^{r}(x)}{g^{m}(x)}{ }_{a} d_{q} x-\int_{a}^{b} \frac{(x-a)^{r}}{g^{m}(x)} f^{r}(x)_{a} d_{q} x .
\end{aligned}
$$

Also, if $q \rightarrow 1$, then (3.13) reduces to the generalization of (1.5), which appeared in [53].

Theorem 3.7 Iff, $g$ are positive functions on $[a, b] \subseteq(0, \infty)$ such that $g$ is non-decreasing, $0<r<1$, and $m>0$, then

$$
\begin{aligned}
& {[r]_{p, q} \int_{a}^{b} \frac{1}{g^{m}(x)}\left(\int_{a}^{x} f(t)_{a} d_{p, q} t\right)_{a}^{r} d_{p, q} x} \\
& \geq(b-a)^{r} \int_{a}^{b} \frac{f^{r}(x)}{g^{m}(b)} a d_{p, q} x-\int_{a}^{b} \frac{(x-a)^{r}}{g^{m}(b)} f^{r}(x)_{a} d_{p, q} x .
\end{aligned}
$$

Proof From Theorem 3.1, we get

$$
\int_{a}^{b} \frac{1}{g^{m}(x)}\left(\int_{a}^{x} f(t)_{a} d_{p, q} t\right)^{r}{ }_{a} d_{p, q} x
$$




$$
\begin{aligned}
\geq & \int_{a}^{b} g^{-m}(x)\left(\int_{a}^{x} f^{r}(t)_{a} d_{p, q} t\right) \\
& \times\left(\int_{a}^{x}{ }_{a} d_{p, q} t\right)^{r-1}{ }_{a} d_{p, q} x \\
= & \int_{a}^{b} g^{-m}(x)\left(\int_{a}^{x} f^{r}(t)_{a} d_{p, q} t\right)(x-a)^{r-1}{ }_{a} d_{p, q} x \\
= & \int_{a}^{b} \int_{t}^{b} g^{-m}(x) f^{r}(t)(x-a)^{r-1}{ }_{a} d_{p, q} x_{a} d_{p, q} t .
\end{aligned}
$$

By the assumption of the function $g$ and Theorem 2.2(v), we obtain

$$
\begin{aligned}
& \int_{a}^{b} \frac{1}{g^{m}(x)}\left(\int_{a}^{x} f(t)_{a} d_{p, q} t\right)^{r}{ }_{a} d_{p, q} x \\
& \quad \geq g^{-m}(b) \int_{a}^{b} f^{r}(t)\left(\int_{t}^{b}(x-a)^{r-1}{ }_{a} d_{p, q} x\right)_{a} d_{p, q} t \\
& \quad=g^{-m}(b) \int_{a}^{b} f^{r}(t)\left(\frac{(b-a)^{r}-(t-a)^{r}}{[r]_{p, q}}\right)_{a} d_{p, q} t \\
& \quad=\frac{1}{[r]_{p, q}}\left((b-a)^{r} \int_{a}^{b} \frac{f^{r}(t)}{g^{m}(b)}{ }_{a} d_{p, q} t-\int_{a}^{b} \frac{(t-a)^{r}}{g^{m}(b)} f^{r}(t)_{a} d_{p, q} t\right) .
\end{aligned}
$$

The proof is accomplished.

Remark 3.6 If $p=1$, then (3.14) reduces to

$$
\begin{aligned}
& {[r]_{q} \int_{a}^{b} \frac{1}{g^{m}(x)}\left(\int_{a}^{x} f(t)_{a} d_{q} t\right)_{a}^{r} d_{q} x} \\
& \quad \geq(b-a)^{r} \int_{a}^{b} \frac{f^{r}(x)}{g^{m}(b)}{ }_{a} d_{q} x-\int_{a}^{b} \frac{(x-a)^{r}}{g^{m}(b)} f^{r}(x)_{a} d_{q} x .
\end{aligned}
$$

Also, if $q \rightarrow 1$, then (3.15) reduces to the generalization of (1.6), which appeared in [53].

Theorem 3.8 Let $f, g$ be positive functions on $[a, b] \subseteq(0, \infty), r \geq 1, m>0$ and

$$
G(x)=\int_{0}^{x} g(t)_{a} d_{p, q} t
$$

If the function $G$ is non-decreasing, then

$$
\begin{aligned}
& {[r]_{p, q} \int_{a}^{b} \frac{1}{G^{m}(x)}\left(\int_{a}^{x} f(t)_{a} d_{p, q} t\right)_{a}^{r} d_{p, q} x} \\
& \quad \leq(b-a)^{r} \int_{a}^{b} \frac{f^{r}(x)}{G^{m}(x)}{ }_{a} d_{p, q} x-\int_{a}^{b} \frac{(x-a)^{r}}{G^{m}(x)} f^{r}(x)_{a} d_{p, q} x .
\end{aligned}
$$

Proof From Theorem 2.3, we get

$$
\int_{a}^{b} \frac{1}{G^{m}(x)}\left(\int_{a}^{x} f(t)_{a} d_{p, q} t\right)_{a}^{r} d_{p, q} x
$$




$$
\leq \int_{a}^{b} G^{-m}(x)\left(\int_{a}^{x} f^{r}(t)_{a} d_{p, q} t\right)\left(\int_{a}^{x}{ }_{a} d_{p, q} t\right)^{r-1}{ }_{a} d_{p, q} x .
$$

By the assumption of the function $G(x)$, we have

$$
\begin{aligned}
& \int_{a}^{b} \frac{1}{G^{m}(x)}\left(\int_{a}^{x} f(t)_{a} d_{p, q} t\right)_{a}^{r} d_{p, q} x \\
& \quad \leq \int_{a}^{b} G^{-m}(x)\left(\int_{a}^{x} f^{r}(t)_{a} d_{p, q} t\right)(x-a)^{r-1}{ }_{a} d_{p, q} x \\
& \quad=\int_{a}^{b} \int_{t}^{b} G^{-m}(x) f^{r}(t)(x-a)^{r-1}{ }_{a} d_{p, q} x_{a} d_{p, q} t \\
& \quad \leq \int_{a}^{b} G^{-m}(t) f^{r}(t)\left(\int_{t}^{b}(x-a)^{r-1}{ }_{a} d_{p, q} x\right)_{a} d_{p, q} t .
\end{aligned}
$$

By Theorem 2.2(v), we obtain

$$
\begin{aligned}
& \int_{a}^{b} \frac{1}{G^{m}(x)}\left(\int_{a}^{x} f(t)_{a} d_{p, q} t\right)^{r}{ }_{a} d_{p, q} x \\
& \quad=\int_{a}^{b} G^{-m}(t) f^{r}(t)\left(\frac{(b-a)^{r}-(t-a)^{r}}{[r]_{p, q}}\right)_{a} d_{p, q} t \\
& \quad=\frac{1}{[r]_{p, q}}\left((b-a)^{r} \int_{a}^{b} \frac{f^{r}(t)}{G^{m}(t)} a_{p, q} t-\int_{a}^{b} \frac{(t-a)^{r}}{G^{m}(t)} f^{r}(t)_{a} d_{p, q} t\right),
\end{aligned}
$$

which gives the required inequality.

Remark 3.7 If $p=1$, then (3.17) reduces to

$$
\begin{aligned}
& {[r]_{q} \int_{a}^{b} \frac{1}{G^{m}(x)}\left(\int_{a}^{x} f(t)_{a} d_{q} t\right)_{a}^{r} d_{q} x} \\
& \quad \leq(b-a)^{r} \int_{a}^{b} \frac{f^{r}(x)}{G^{m}(x)}{ }_{a} d_{q} x-\int_{a}^{b} \frac{(x-a)^{r}}{G^{m}(x)} f^{r}(x)_{a} d_{q} x .
\end{aligned}
$$

Also, if $q \rightarrow 1$, then (3.19) reduces to the generalization of (1.5).

Theorem 3.9 Let $f, g$ be positive functions on $[a, b] \subseteq(0, \infty), 0<r<1, m>0$ and let $G(x)$ be defined by (3.16). If the function $G$ is non-decreasing, then

$$
\begin{aligned}
{[r]_{p, q} \int_{a}^{b} \frac{1}{G^{m}(x)}\left(\int_{a}^{x} f(t)_{a} d_{p, q} t\right)^{r} d_{p, q} x \geq } & (b-a)^{r} \int_{a}^{b} \frac{f^{r}(x)}{G^{m}(b)}{ }_{a} d_{p, q} x \\
& -\int_{a}^{b} \frac{(x-a)^{r}}{G^{m}(b)} f^{r}(x)_{a} d_{p, q} x .
\end{aligned}
$$

Proof From Theorem 3.1, we get

$$
\begin{aligned}
\int_{a}^{b} \frac{1}{G^{m}(x)}\left(\int_{a}^{x} f(t)_{a} d_{p, q} t\right)^{r}{ }_{a} d_{p, q} x \geq & \int_{a}^{b} G^{-m}(x)\left(\int_{a}^{x} f^{r}(t)_{a} d_{p, q} t\right) \\
& \times\left(\int_{a}^{x}{ }_{a} d_{p, q} t\right)^{r-1}{ }_{a} d_{p, q} x .
\end{aligned}
$$


By the assumption of the function $G(x)$, we have

$$
\begin{aligned}
& \int_{a}^{b} \frac{1}{G^{m}(x)}\left(\int_{a}^{x} f(t)_{a} d_{p, q} t\right)_{a}^{r} d_{p, q} x \\
& \geq \int_{a}^{b} G^{-m}(x)\left(\int_{a}^{x} f^{r}(t)_{a} d_{p, q} t\right)(x-a)^{r-1}{ }_{a} d_{p, q} x \\
& \quad=\int_{a}^{b} \int_{t}^{b} x^{m} G^{-m}(x) f^{r}(t)(x-a)^{r-1}{ }_{a} d_{p, q} x_{a} d_{p, q} t \\
& \geq \int_{a}^{b} G^{-m}(b) f^{r}(t)\left(\int_{t}^{b}(x-a)^{r-1}{ }_{a} d_{p, q} x\right)_{a} d_{p, q} t .
\end{aligned}
$$

By Theorem 2.2(v), we obtain

$$
\begin{aligned}
& \int_{a}^{b} \frac{1}{G^{m}(x)}\left(\int_{a}^{x} f(t)_{a} d_{p, q} t\right)^{r}{ }_{a} d_{p, q} x \\
& \quad=\int_{a}^{b} G^{-m}(b) f^{r}(t)\left(\frac{(b-a)^{r}-(t-a)^{r}}{[r]_{p, q}}\right)_{a} d_{p, q} t \\
& \quad=\frac{1}{[r]_{p, q}}\left((b-a)^{r} \int_{a}^{b} \frac{f^{r}(t)}{G^{m}(b)}{ }_{a} d_{p, q} t-\int_{a}^{b} \frac{(t-a)^{r}}{G^{m}(b)} f^{r}(t)_{a} d_{p, q} t\right)
\end{aligned}
$$

provided the left and right sides are finite.

Remark 3.8 If $p=1$, then (3.20) reduces to

$$
\begin{aligned}
& {[r]_{q} \int_{a}^{b} \frac{1}{G^{m}(x)}\left(\int_{a}^{x} f(t)_{a} d_{q} t\right)^{r}{ }_{a} d_{q} x} \\
& \quad \geq(b-a)^{r} \int_{a}^{b} \frac{f^{r}(x)}{G^{m}(b)}{ }_{a} d_{q} x-\int_{a}^{b} \frac{(x-a)^{r}}{G^{m}(b)} f^{r}(x)_{a} d_{q} x .
\end{aligned}
$$

Also, if $q \rightarrow 1$, then (3.22) reduces to the generalization of (1.6).

\section{Examples}

In the following, we will give examples to illustrate our main results.

Example 4.1 Define the continuous functions $f, g:[1,3] \rightarrow \mathbb{R}$ by $f(x)=x^{4}$ and $g(x)=1 / x$. Applying Theorem 3.1 with $p=2 / 3, q=1 / 3, r=1 / 2$ and $s=-1$, the left side of (3.1) becomes

$$
\begin{aligned}
\int_{a}^{b}|f(x) g(x)|_{a} d_{p, q} x & =\int_{1}^{3}\left|x^{4}(1 / x)\right|_{1} d_{2 / 3,1 / 3} x \\
& =\int_{1}^{3} x^{3}{ }_{1} d_{2 / 3,1 / 3} x \\
& =\frac{(3-1)^{4}}{[4]_{2 / 3,1 / 3}}+\frac{3(1)(3-1)^{3}}{[3]_{2 / 3,1 / 3}}+\frac{3(1)^{2}(3-1)^{2}}{[2]_{2 / 3,1 / 3}}+1^{3}(3-1) \\
& \approx 73.65714286 .
\end{aligned}
$$


For the right side of (3.1), one has

$$
\begin{aligned}
& \left(\int_{a}^{b}|f(x)|^{r}{ }_{a} d_{p, q} x\right)^{1 / r}\left(\int_{a}^{b}|g(x)|_{a}^{s} d_{p, q} x\right)^{1 / s} \\
& \quad=\left(\int_{1}^{3}\left(x^{4}\right)^{1 / 2}{ }_{1} d_{2 / 3,1 / 3} x\right)^{2}\left(\int_{1}^{3}(1 / x)^{-1}{ }_{1} d_{2 / 3,1 / 3} x\right)^{-1} \\
& \quad=\left(\int_{1}^{3} x^{2}{ }_{1} d_{2 / 3,1 / 3} x\right)^{2}\left(\int_{1}^{3} x_{1} d_{2 / 3,1 / 3} x\right)^{-1} \\
& \quad=\left[\frac{(3-1)^{3}}{[3]_{2 / 3,1 / 3}}+\frac{2(1)(3-1)^{2}}{[2]_{2 / 3,1 / 3}}+1^{2}(3-1)\right]^{2}\left[\frac{(3-1)[3-1(1-2 / 3-1 / 3)]}{[2]_{2 / 3,1 / 3}}\right]^{-1} \\
& \quad \approx 68.58503401 .
\end{aligned}
$$

It is clear that $73.65714286 \geq 68.58503401$, which confirms the result described in Theorem 3.1 .

Example 4.2 Define functions $f, g:[1,3] \rightarrow \mathbb{R}$ by $f(x)=x$ and $g(x)=x-1 / 6$. Then $f$ is non-negative function and $g$ is a positive function on $[1,3]$. Applying Theorem 3.3 with $p=1 / 2, q=1 / 3, r=m=2$ and $\gamma=5 / 6$, the left side of (3.6) becomes

$$
\begin{aligned}
\int_{a}^{b} \frac{1}{g^{m}(x)}\left(\int_{a}^{x} f(t)_{a} d_{p, q} t\right)_{a}^{r} d_{p, q} x & =\int_{1}^{3} \frac{1}{(x-1 / 6)^{2}}\left(\int_{1}^{x} t_{1} d_{1 / 2,1 / 3} t\right)^{2}{ }_{1} d_{1 / 2,1 / 3} x \\
& =\int_{1}^{3} \frac{1}{(x-1 / 6)^{2}} \frac{(x-1)^{2}(x-1 / 6)^{2}}{(5 / 6)^{2}}{ }_{1} d_{1 / 2,1 / 3} x \\
& =\frac{36}{25} \frac{(3-1)^{3}}{[2+1]_{1 / 2,1 / 3}} \\
& =\frac{10,368}{475} \\
& \approx 21.82736842
\end{aligned}
$$

by Theorem $2.2(\mathrm{v})$.

For the right side of (3.6), one has

$$
\begin{aligned}
& \frac{(p q)^{m+1-r-1 / r}}{[1-1 / r]_{p, q}^{r-1}[m+1-r-1 / r]_{p, q}} \int_{a}^{b} \frac{(x-a+\gamma)^{r}}{g^{m}(x)} f^{r}(x)_{a} d_{p, q} x \\
& =\frac{(1 / 6)^{2+1-2-1 / 2}}{[1-1 / 2]_{1 / 2,1 / 3}^{2-1}[2+1-2-1 / 2]_{1 / 2,1 / 3}} \int_{1}^{3} \frac{(x-1+5 / 6)^{2}}{(x-1 / 6)^{2}} x^{2}{ }_{1} d_{1 / 2,1 / 3} x \\
& =\frac{(1 / 6)^{1 / 2}}{[1 / 2]_{1 / 2,1 / 3}^{2}} \int_{1}^{3} x^{2}{ }_{1} d_{1 / 2,1 / 3} x \\
& =\frac{(1 / 6)^{1 / 2}}{[1 / 2]_{1 / 2,1 / 3}^{2}}\left[\frac{(3-1)^{3}}{[3]_{1 / 2,1 / 3}}+\frac{2(3-1)^{2}}{[2]_{1 / 2,1 / 3}}+1^{2}(3-1)\right] \\
& \approx 40.46697790 .
\end{aligned}
$$

It is clear that $21.82736842 \leq 40.46697790$, which confirms the result described in Theorem 3.3. 


\section{Conclusion}

In the present paper, we use $(p, q)$-calculus to establish new integral inequalities related to Hardy type integral inequalities. Many existing results in the literature are reduced to special cases of our results when $p=1$ and $q \rightarrow 1$. The results of this paper are new and significantly contribute to the existing literature on the topic. In addition, we shall study these results in fractional $(p, q)$-calculus and conformable fractional $(p, q)$-calculus in the future.

\section{Acknowledgements \\ This work is supported by Science Achievement Scholarship of Thailand, SAST and the Program Management Unit for Human Resources \& Institutional Development, Research and Innovation [grant number B05F630104].}

\section{Funding}

Not applicable.

\section{Availability of data and materials}

Not applicable.

\section{Competing interests}

The authors declare that they have no competing interests.

\section{Authors' contributions}

All authors contributed equally to this article. They read and approved the final manuscript.

\section{Author details}

${ }^{1}$ Department of Mathematics, Khon Kaen University, 40002, Khon Kaen, Thailand. ${ }^{2}$ Department of Mathematics, Faculty of Applied Science, King Mongkut's University of Technology North Bangkok, 10800, Bangkok, Thailand. ${ }^{3}$ Department of Mathematics, University of loannina, 45110, loannina, Greece. ${ }^{4}$ Nonlinear Analysis and Applied Mathematics (NAAM)-Research Group, Department of Mathematics, Faculty of Science, King Abdulaziz University, 21588, Jeddah, Saudi Arabia.

\section{Publisher's Note}

Springer Nature remains neutral with regard to jurisdictional claims in published maps and institutional affiliations.

Received: 20 February 2021 Accepted: 6 June 2021 Published online: 12 June 2021

\section{References}

1. Jackson, F.H.: On a q-definite integrals. Q. J. Pure Appl. Math. 41, 193-203 (1910)

2. Jackson, F.H.: q-difference equations. Am. J. Math. 32, 305-314 (1910)

3. Kac, V., Cheung, P.: Quantum Calculus. Springer, New York (2002)

4. Bangerezako, G.: Variational calculus on q-nonuniform lattices. J. Math. Anal. Appl. 306, 161-179 (2005)

5. Exton, H.: q-Hypergeomatric Functions and Applications. Hastead Press, New York (1983)

6. Tariboon, J., Ntouyas, S.K.: Quantum calculus on finite intervals and applications to impulsive difference equations. Adv. Differ. Equ. 2013, 282 (2013)

7. Annaby, M.H., Mansour, Z.S.: q-Fractional Calculus and Equations. Springer, Heidelberg (2012)

8. Ernst, T.: A Comprehensive Treatment of $q$-Calculus. Springer, Basel (2012)

9. Ernst, T:: The history of $q$-calculus and a new method. UUDM Report 2000:16, Department of Mathematics, Uppsala University, (2000)

10. Noor, M.A., Awan, M.U., Noor, K.I.: Quantum Ostrowski inequalities for q-differentiable convex functions. J. Math Inequal. 10, 1013-1018 (2016)

11. Aral, A., Gupta, V., Agarwal, R.P.: Applications of q-Calculus in Operator Theory. Springer, Berlin (2013)

12. Gauchman, H.: Integral inequalities in q calculus. Comput. Math. Appl. 47, 281-300 (2004)

13. Erden, S., Iftikhar, S., Kumam, P., Awan, M.U.: Some Newton's like inequalities with applications. Rev. R. Acad. Cienc. Exactas Fís. Nat., Ser. A Mat. 114, 195 (2020)

14. Erden, S., Budak, H., Sarikaya, M.Z., Iftikhar, S., Kumam, P.: Fractional Ostrowski type inequalities for bounded functions. J. Inequal. Appl. 2020, 123 (2020)

15. Erden, S., Iftikhar, S., Delavar, M.R., Kumam, P., Thounthong, P., Kumam, W.: On generalizations of some inequalities for convex functions via quantum integrals. Rev. R. Acad. Cienc. Exactas Fís. Nat., Ser. A Mat. 114, 110 (2020)

16. Iftikhar, S., Kumam, P., Erden, S.: Newton's-type integral inequalities via local fractional integrals. Fractals 28, 3 (2020)

17. Sadjang, P.N.: On the fundamental theorem of $(p, q)$-calculus and some $(p, q)$-Taylor formulas. Results Math. 73, 1-21 (2013)

18. Tunç, M., Göv, E.: Some integral inequalities via $(p, q)$-calculus on finite intervals. RGMIA Res. Rep. Collect. 95, 1-12 (2016)

19. Chakrabarti, R., Jagannathan, R.: A $(p, q)$-oscillator realization of two-parameter quantum algebras. J. Phys. A, Math Gen. 24, L711-L718 (1991) 
20. Burban, I.M., Klimyk, A.U.: $(p, q)$-differentiation, $(p, q)$-integration and $(p, q)$-hypergeometric functions related to quantum groups. Integral Transforms Spec. Funct. 2, 15-36 (1994)

21. Hounkonnou, M.N., Kyemba, J.D.B.: R(p,q)-calculus: differentiation and integration. SUT J. Math. 49, 145-167 (2013)

22. Aral, A., Gupta, V.: Applications of $(p, q)$-gamma function to Szasz Durrmeyer operators. Publ. Inst. Math. 102(116), $211-220(2017)$

23. Sahai, V., Yadav, S.: Representations of two parameter quantum algebras and $(p, q)$-special functions. J. Math. Anal. Appl. 335, 268-279 (2007)

24. Tunç, M., Göv, E.: (p,q)-integral inequalities. RGMIA Res. Rep. Collect. 97, 1-13 (2016)

25. Tunç, M., Göv, E.: (p,q)-integral inequalities for convex functions. RGMIA Res. Rep. Collect. 98, 1-12 (2016)

26. Prabseang, J., Nonlaopon, K., Tariboon, J.: (p,q)-Hermite-Handamard inequalities for double integral and $(p, q)$-differentiable convex function. Axioms 8(2), 68 (2019)

27. Kunt, M., Iscan, I., Alp, N., Sarikaya, M.Z.: $(p, q)$-Hermite-Hadamard inequalities and (p, q)-estimates for midpoint type inequalities via convex and quasi-convex functions. Rev. R. Acad. Cienc. Exactas Fís. Nat., Ser. A Mat. 112, 969-992 (2018)

28. Sadjang, P.N.: On the $(p, q)$-Gamma and the $(p, q)$-Beta functions. (2015). arXiv:1506.07394

29. Kalsoom, H., Amer, M., Junjua, M.D., Hassain, S., Shahzadi, G.: Some (p, q)-estimates of Hermite-Hardamard-type inequalities for coordinated convex and quasi convex functions. Mathematics 7(8), 683 (2019)

30. Latif, M.A., Kunt, M., Dragomir, S.S., Iscan, I.: Post-quantum trapezoid type inequalities. AIMS Math. 5, 4011-4026 (2020)

31. Li, C., Yang, D., Bai, C.: Some Opial type inequalities in (p, q)-calculus. AlMS Math. 5, 5893-5902 (2020)

32. Hardy, G.H.: Inequalities. Cambridge University Press, Cambridge (1967)

33. Skrzypczak, l.: Hardy-type inequalities derived from p-harmonic problems. Nonlinear Anal. 93, 30-50 (2013)

34. Dubinskii, Y.A.: A Hardy-type inequality and its applications. Proc. Steklov Inst. Math. 269, 106-126 (2010)

35. Bicheng, Y.: On Hardy's inequality. J. Math. Anal. Appl. 234, 717-722 (1999)

36. Kufner, A., Maligranda, L., Persson, L.E.: The prehistory of the Hardy inequality. Am. Math. Mon. 113, 715-732 (2006)

37. Bougoffa, L.: On Minkowski and Hardy integral inequalities. J. Inequal. Pure Appl. Math. 7(2), 60 (2006)

38. Duy, N.T.: Some variants of the Hardy inequality. Appl. Anal. (2020). https://doi.org/10.1080/00036811.2020.1828370

39. Osekowski, A.: A new approach to Hardy-type inequalities. Arch. Math. 104, 165-176 (2015)

40. Maligranda, L., Oinarov, R., Persson, L.E.: On Hardy q-inequalities. Czechoslov. Math. J. 64, 659-682 (2014)

41. Persson, L.E., Shaimardan, S.: Some new Hardy-type inequalities for Riemann-Liouville fractional $q$-integral operator. J. Inequal. Appl. 296, 1-17 (2015)

42. Shaimardan, S.: Hardy-type inequalities for the fractional integral operator in q-analysis. Eurasian Math. J. 7, 84-99 (2016)

43. Baiarystanov, A.O., Persson, L.E., Shaimardan, S., Termirkhanova, A.: Some new Hardy-type inequalities in $q$-analysis. J. Math. Inequal. 3, 761-781 (2016)

44. Levinson, N.: Generalization of inequalities of Hardy. Duke Math. J. 31, 389-394 (1964)

45. Sulaiman, W.T.: Some Hardy type integral inequalities. Appl. Math. Lett. 25, 520-525 (2012)

46. Sroysang, B.: A generalization of some integral inequalities similar to Hardy's inequality. Math. Æterna 3, 593-596 (2013)

47. Sroysang, B.: More on some Hardy type integral inequalities. J. Math. Inequal. 8, 497-501 (2014)

48. Khaled, M.: Some generalizations and refined Hardy type integral inequalities. Afr. Math. 28, 451-457 (2017)

49. Sulaiman, W.T.: Revesrses of Minkowski's, Holder's, and Hardy's integral inequalities. Int. J. Mod. Math. Sci. 1, 4-24 (2012)

50. Saker, S.H., Mahmoud, R.R., Osman, M.M., Agarwal, R.P.: Some new generalized forms of Hardy's type inequality on time scales. Math. Inequal. Appl. 20,459-481 (2017)

51. Ajisope, M.O., Rauf, K.: On some refinements of Hardy-type integral inequalities. Int. J. Math. Comput. 14, 879-888 (2019)

52. Dahmani, Z., Khameli, A., Freha, K.: Further generalizations on some Hardy type RL-integral inequalities. J. Int. Math. (2020). https://doi.org/10.1080/09720502.2020.1754543

53. Wu, S., Sroysang, B., Li, S.: A further generalization of certain integral inequalities similar to Hardy's inequality. J. Nonlinear Sci. Appl. 9, 1093-1102 (2018)

\section{Submit your manuscript to a SpringerOpen ${ }^{\circ}$ journal and benefit from:}

- Convenient online submission

- Rigorous peer review

- Open access: articles freely available online

- High visibility within the field

- Retaining the copyright to your article

Submit your next manuscript at $\boldsymbol{s p r i n g e r o p e n . c o m ~}$ 